American Journal of Applied Sciences 6 (1): 1-12, 2009

ISSN 1546-9239

(C) 2009 Science Publications

\title{
Multi-Product Multi-Constraint Inventory Control Systems with Stochastic Replenishment and Discount under Fuzzy Purchasing Price and Holding Costs
}

\author{
${ }^{1}$ Ata Allah Taleizadeh, ${ }^{2}$ Seyed Taghi Akhavan Niaki and ${ }^{1}$ Mir-Bahador Aryanezhad \\ ${ }^{1}$ Department of Industrial Engineering, Iran University of Science and Technology, Iran \\ ${ }^{2}$ Department of Industrial Engineering, Sharif University of Technology, Iran
}

\begin{abstract}
While in multi-periodic inventory control problems the usual assumption are that the orders are placed at the beginning of each period (periodic review) or depending on the inventory level they can happen at any time (continuous review), in this research, we relax these assumptions and assume that the periods between two replenishments of the products are independent and identically distributed random variables. Furthermore, assuming the purchasing price are triangular fuzzy variables, the quantities of the orders are of integer-type and that there are space, budget and service level constraints, incremental discount is considered to purchase products and a combination of back-order and lost-sales are taken into account for the shortages. We show that the model of this problem is a fuzzy mixed-integer nonlinear programming type and in order to solve it, a hybrid method of fuzzy simulation and genetic algorithm approach is used. At the end, a numerical example is given to demonstrate the applicability of the proposed methodology in real world inventory control problems.
\end{abstract}

Key words: Inventory control, stochastic replenishment, discount, fuzzy mixed-integer nonlinear programming, fuzzy simulation, genetic algorithm

\section{INTRODUCTION}

In multi-periodic inventory control models, the continuous review and the periodic review are the major vastly used policies. However, the underlying assumptions of the proposed models restrict their correct usage and utilization in real-world environments. In continuous review policy, the user has the freedom to act at anytime and replenish orders based upon the available inventory level. While in the periodic review policy, the user is allowed to replenish the orders only in specific and predetermined times.

The multi-periodic inventory control problems have been investigated in depth in different research. Chiang ${ }^{[1]}$ considered a periodic review model in which the period is partly long. The important aspect of his study was to introduce emergency orders to prevent shortages. He employed a dynamic programming approach to model the problem. Mohebbi and Posner ${ }^{[2]}$ investigated an inventory system with periodic review, multiple replenishment and multi-level delivery. They assumed that the stochastic demand followed Poisson distribution, shortages were allowed and that the lost sale policy could be employed. Feng and $\mathrm{RaO}^{[3]}$ considered a $(\mathrm{R}, \mathrm{nT})$ model in which in the first level a stochastic demand entered the system and the total unsatisfied demand were back-ordered at the second level. Ouyang and Chuang $^{[4]}$ investigated a $(R, T)$ model in which the period-length and lead-time were the decision variables, demand was a random variable and the service level was a constraint. Chiang ${ }^{[5]}$ analyzed a periodic review problem in two cases of back-order and lost sales and employed the (R, T) policy. $\mathrm{Qu}$ et al ${ }^{[6]}$ investigated a transportation model integrated with an inventory model with a periodic review policy. Eynan and Kropp ${ }^{[7]}$ have propounded the assumption of stochastic demand and variant warehousing costs on a periodic review system, while assuming nonzero lead-time and safety stock. Bylka ${ }^{[8]}$ investigated a model with constraints on the amounts of orders and back-order shortages in which the lead-time was constant and demand was stochastic and Mohebbi ${ }^{[9]}$ considered demand a compound Poisson random variable. Furthermore, Taleizadeh et al. ${ }^{[10]}$ investigated a stochastic replenishment multi-product inventory system and proposed two models for two cases of uniform and exponential distribution of the time between two replenishments. They showed that the models were of integer non-linear type and proposed a simulation annealing algorithm to solve them.

Corresponding Author: Seyed Taghi Akhavan Niaki, Department of Industrial Engineering, Sharif University of Technology, Iran 
In the literature review of the fuzzy inventory models, Hsieh ${ }^{[11]}$ introduced two models with fuzzy parameters for crisp and fuzzy production quantities. In Roy et $a l .{ }^{[12]}$ research an inventory model for a deteriorating item with stock dependent demand was developed under two storage facilities over a random planning horizon. Then, for the crisp deterioration rate on one hand, the expected profit was derived and maximized via Genetic Algorithm (GA). On the other hand, when deterioration rate was imprecise, the optimistic/pessimistic equivalent of fuzzy objective function was obtained using possibility/necessity measure of fuzzy event. Yao et al. ${ }^{[13]}$ considered an inventory problem without backorder where both the order and the total demand quantities were triangular fuzzy numbers. Das et al. ${ }^{[14]}$ formulated multi-item stochastic and fuzzy-stochastic inventory problems under total budgetary and space constraints. Chang et al. ${ }^{[15]}$ investigated the fuzzy problems of the mixture inventory systems involving variable lead-time with backorders and lost sales. In order to maximize the average profit, Mandal and Roy ${ }^{[16]}$ formulated a multiitem displayed inventory problem under shelf-space constraint in a fuzzy environment. In another research in this area, Maiti and Maiti ${ }^{[17]}$ developed a multi-item inventory model with two-storage facilities and advertisement where the price and displayed inventory level-dependent demand, the purchase cost, the investment amount and storehouse capacity were imprecise. The problem was formulated as a single/multi-objective programming problem under fuzzy constraint. Liu ${ }^{[18]}$ developed a solution method to derive the fuzzy profit of the inventory model when the demand quantity and the unit cost were fuzzy numbers.

Five main specifications of the proposed model of this research that have led to its novelty are the stochastic period length, the allowance of multiproducts multi-constraint, the purchasing price being fuzzy variable, incorporating discounts to purchase products and the fact that the decision variables are integer. By deploying these conditions simultaneously, the problem becomes more realistic and the created model is different from the other models in the periodic review literature.

\section{A BRIEF BACKGROUND IN FUZZY ENVIRONMENT}

In this research, we adopt the concepts of the credibility theory including possibility, necessity and credibility of fuzzy event and the expected value of a fuzzy variable defined as ${ }^{[19]}$ :
Definition 1: Let $\xi$ be a fuzzy variable with the membership function $\mu(x)$. Then the possibility, necessity and credibility measure of the fuzzy event $\xi \geq \mathrm{r}$ can be represented, respectively, by:

$$
\begin{gathered}
\operatorname{Pos}\{\xi>r\}=\sup _{\mathrm{u} \geq \mathrm{r}} \mu(\mathrm{u}) \\
\operatorname{Nec}\{\xi \geq \mathrm{r}\}=1-\sup _{\mathrm{u}<\mathrm{r}} \mu(\mathrm{u}) \\
\operatorname{Cr}\{\xi \geq \mathrm{r}\}=\frac{1}{2}[\operatorname{Pos}\{\xi \geq \mathrm{r}\}+\operatorname{Nec}\{\xi \geq \mathrm{r}\}]
\end{gathered}
$$

Definition 2: The expected value of a fuzzy variable is defined as:

$$
\mathrm{E}[\xi]=\int_{0}^{\infty} \operatorname{Cr}\{\xi \geq \mathrm{r}\} \mathrm{dr}-\int_{-\infty}^{0} \operatorname{Cr}\{\xi \leq \mathrm{r}\} \mathrm{dr}
$$

Definition 3: The optimistic function of $\alpha$ is defined as:

$$
\xi_{\text {sup }}(\alpha)=\sup [\mathrm{r} \mid \operatorname{Cr}\{\xi \geq \mathrm{r}\} \geq \alpha], \quad \alpha \in(0,1]
$$

\section{PROBLEM DEFINITION}

Consider a periodic inventory control model for one provider in which the times required to order each of several available products are stochastic in nature. Let the time-periods between two productreplenishments be identical and independent random variables; the purchasing price of the products to be triangular fuzzy variables, the demands are crisp and in case of shortage, a fraction are considered back-order and a fraction as lost-sale. The costs associated with the inventory control system are holding (a percentage of the purchasing cost), back-order, lost-sales and purchasing costs. Furthermore, the incremental discount policy is used, the service level of each product, warehouse space and budget are considered constraints of the problem and the decision variables are integer digits. We need to identify the inventory levels in each cycle such that the expected profit is maximized.

\section{PROBLEM MODELING}

For the problem at hand, since the time-periods between two replenishments are independent random variables, in order to maximize the expected profit of the planning horizon we need to consider only one period. Furthermore, since we assumed that the costs 
associated with the inventory control system are holding and shortage (back-order and lost-sale), we need to calculate the expected inventory level and the expected required storage space in each period. Before doing this, let us define the parameters and the variables of the model.

The parameters and the variables of the model: For $\mathrm{i}=1,2, \ldots, \mathrm{n}$, let us define the parameters and the variables of the model as:

$\mathrm{R}_{\mathrm{i}}$ : $\quad$ The inventory level of the $\mathrm{i}^{\text {th }}$ product

$\mathrm{T}_{\mathrm{i}}$ : $\quad$ A random variable denoting the time-period between two replenishments (cycle length) of the $i^{\text {th }}$ product

$\mathrm{F}_{\mathrm{Ti}}\left(\mathrm{t}_{\mathrm{i}}\right)$ : The Probability density function of $\mathrm{T}_{\mathrm{i}}$

$\mathrm{q}_{\mathrm{ij}}$ : $\quad$ The $\mathrm{j}^{\text {th }}$ discount point for the $\mathrm{i}^{\text {th }}$ product

$\mathrm{W}_{\mathrm{i}}$ : $\quad$ The crisp purchasing cost per unit of the $i^{\text {th }}$ product without discount

$\mathrm{W}_{\mathrm{ij}}$ : The crisp purchasing cost per unit of the $i^{\text {th }}$ product at the $\mathrm{j}^{\text {th }}$ discount point

$\tilde{\mathrm{W}}_{\mathrm{ij}}$ : $\quad$ The fuzzy purchasing cost per unit of the $i^{\text {th }}$ product at the $\mathrm{j}^{\text {th }}$ discount point

$\overline{\mathrm{W}}_{\mathrm{i}}$ : $\quad$ The weighted expected purchasing cost of the $i^{\text {th }}$ product

$\mathrm{FI}_{\mathrm{i}}$ : $\quad$ A fraction of the purchasing cost of the $i^{\text {th }}$ product used to calculate its holding cost

$\mathrm{h}_{\mathrm{i}}$ : $\quad$ The holding cost per unit inventory of the $\mathrm{i}^{\text {th }}$ product in each period

$\mathrm{h}_{\mathrm{i}}^{\prime}$ : The crisp holding cost per unit inventory of the $\mathrm{i}^{\text {th }}$ product in each period $\left(\mathrm{h}_{\mathrm{i}}^{\prime}=\mathrm{FI}_{\mathrm{i}} * \overline{\mathrm{W}}_{\mathrm{i}}\right)$

$\widetilde{\mathrm{h}_{\mathrm{i}}^{\prime}}$ : The fuzzy holding cost per unit inventory of the $\mathrm{i}^{\text {th }}$ product in each period $\left(\widetilde{\mathrm{h}_{\mathrm{i}}^{\prime}}=\mathrm{FI}_{\mathrm{i}} * \overline{\mathrm{W}}_{\mathrm{i}}\right)$

$\mathrm{Q}_{\mathrm{ij}}$ : $\quad$ The order quantity of the $\mathrm{i}^{\text {th }}$ product at the $\mathrm{j}^{\text {th }}$ discount price

$\pi_{\mathrm{i}}$ : The back-order cost per unit demand of the $\mathrm{i}^{\text {th }}$ product

$\hat{\pi}_{\mathrm{i}}$ : The shortage cost for each unit of lost sale of the $i^{\text {th }}$ product

$\mathrm{P}_{\mathrm{i}}$ : $\quad$ The sale price per unit of the $\mathrm{i}^{\text {th }}$ product

$\mathrm{D}_{\mathrm{i}}$ : $\quad$ The constant demand rate of the $\mathrm{i}^{\text {th }}$ product

$\mathrm{SL}_{\mathrm{i}}$ : $\quad$ The lower limit of the service level for the $i^{\text {th }}$ product

$t_{\mathrm{Di}}$ : The time at which the inventory level of the $\mathrm{i}^{\text {th }}$ product reaches zero

$\beta_{\mathrm{i}}$ : $\quad$ The percentage of unsatisfied demands of the $\mathrm{i}^{\text {th }}$ product that is back-ordered

$\mathrm{I}_{\mathrm{i}}$ : $\quad$ The expected amount of the $i^{\text {th }}$ product inventory per cycle
$\mathrm{L}_{\mathrm{i}}$ : The expected amount of the $\mathrm{i}^{\text {th }}$ product lostsale in each cycle.

$\mathrm{B}_{\mathrm{i}}$ : $\quad$ The expected amount of the $\mathrm{i}^{\text {th }}$ product backorder in each cycle

$\mathrm{Q}_{\mathrm{i}}$ : $\quad$ The expected amount of the $\mathrm{i}^{\text {th }}$ product order in each cycle

$\mathrm{f}_{\mathrm{i}}$ : $\quad$ The required warehouse space per unit of the $\mathrm{i}^{\text {th }}$ product

F: $\quad$ Total available warehouse space

TB: $\quad$ Total available budget

$\mathrm{C}_{\mathrm{hi}}$ : $\quad$ The expected holding cost per cycle of the $i^{\text {th }}$ product.

$\mathrm{C}_{\mathrm{bi}}$ : The expected shortage cost in back-order state of the $i^{\text {th }}$ product

$\mathrm{C}_{\mathrm{l}_{\mathrm{i}}}$ : The expected shortage cost in lost-sale state of the $\mathrm{i}^{\text {th }}$ product

$\mathrm{C}_{\mathrm{pi}}$ : $\quad$ The expected purchase cost of the $\mathrm{i}^{\text {th }}$ product

$\mathrm{r}_{\mathrm{i}}$ : $\quad$ The expected revenue obtained from sales

$\mathrm{Z}(\mathrm{R}, \tilde{\mathrm{W}}, \tilde{\mathrm{h}})$ : The expected profit obtained in each cycle

For sake of simplicity, we first consider a singleproduct problem in which the purchasing prices and holding costs are crisp and there is no discount. Then, we are devoted for a single-product problem with incremental and total discount policies, respectively. We discuss the cases in which the demands are fuzzy random variables. Finally, we extend the single-product to the multi-product modeling. However, let us introduce the pictorial representation of the singleproduct problem.

Inventory diagram: According to Ertogal and Rahim $^{[20]}$ and considering the fact that the time-periods between replenishments are stochastic variables, two cases may occur. In the first case the time-period between replenishments is less than the amount of time required for the inventory level to reach zero (Fig. 1) and in the second case, it is greater (Fig. 2).

Single product model-back order and lost sales cases: In this section, we first model the costs, the profit and the constraint of a single-product inventory problem with crisp demand where there is no discount on purchasing products. The replenishments are stochastic and back-order and lost-sales are allowed.

Calculating the costs and the profit: In order to calculate the expected profit in each cycle, we need to evaluate all of the terms in Eq. $6^{[20]}$ :

$$
\begin{aligned}
\mathrm{Z}_{\mathrm{i}}= & \mathrm{r}_{\mathrm{i}}-\mathrm{C}_{\mathrm{p}_{\mathrm{i}}}-\mathrm{C}_{\mathrm{h}_{\mathrm{i}}}-\mathrm{C}_{\mathrm{b}_{\mathrm{i}}}-\mathrm{C}_{\mathrm{l}_{\mathrm{i}}}=\mathrm{P}_{\mathrm{i}} \mathrm{Q}_{\mathrm{i}} \\
& -\mathrm{W}_{\mathrm{i}} \mathrm{Q}_{\mathrm{i}}-\mathrm{h}_{\mathrm{i}} \mathrm{I}_{\mathrm{i}}-\pi_{\mathrm{i}} \mathrm{B}_{\mathrm{i}}-\hat{\pi}_{\mathrm{i}} \mathrm{L}_{\mathrm{i}}
\end{aligned}
$$




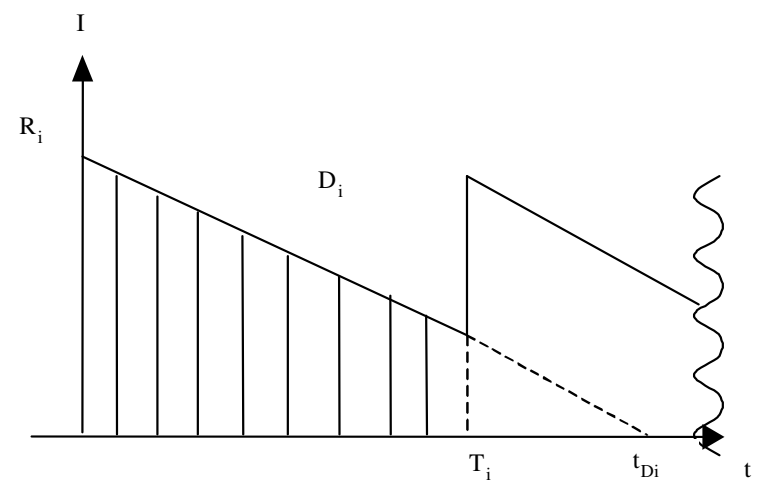

Fig. 1: Presenting the inventory cycle when $\mathrm{T}_{\operatorname{Min}_{\mathrm{i}}} \leq \mathrm{T}_{\mathrm{i}} \leq \mathrm{t}_{\mathrm{D}_{\mathrm{i}}}$

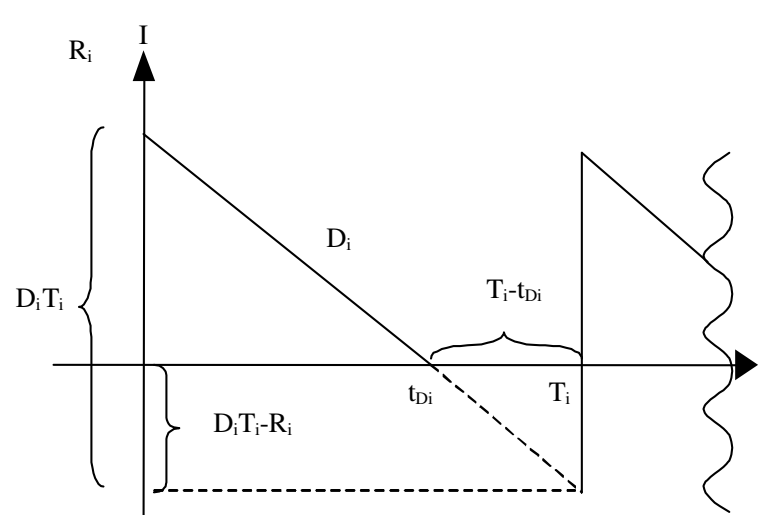

Fig. 2: Presenting the inventory cycle when $t_{D_{i}}<T_{i} \leq T_{M^{2} x_{i}}$

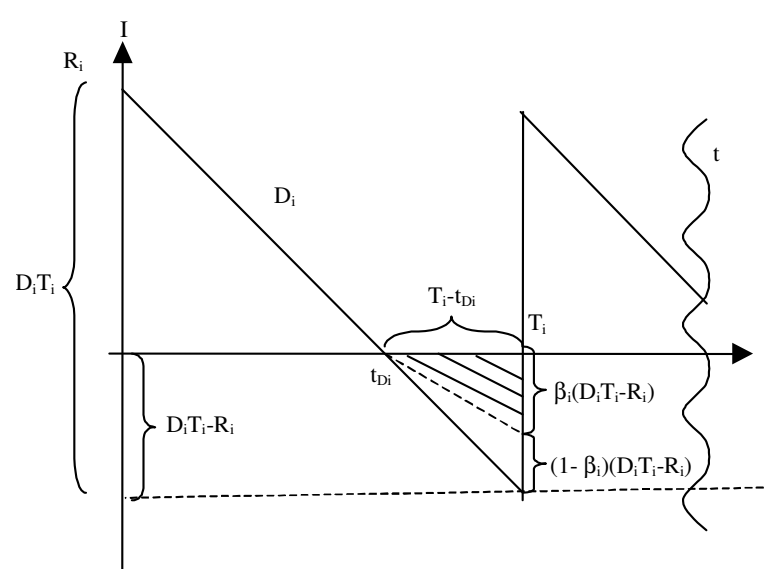

Fig. 3: Presenting shortages in two cases of back order and lost sales

Based on Fig. 3, $\mathrm{L}_{\mathrm{i}}, \mathrm{B}_{\mathrm{i}}, \mathrm{I}_{\mathrm{i}}$ and $\mathrm{Q}_{\mathrm{i}}$ are evaluated by the following equations:

$$
\begin{aligned}
& \mathrm{L}_{\mathrm{i}}=\left(1-\beta_{\mathrm{i}}\right) \int_{\mathrm{t}_{\mathrm{D}_{\mathrm{i}}}}^{\mathrm{T}_{\mathrm{Max}_{\mathrm{i}}}}\left(\mathrm{D}_{\mathrm{i}} \mathrm{T}_{\mathrm{i}}-\mathrm{R}_{\mathrm{i}}\right) \mathrm{f}_{\mathrm{T}_{\mathrm{i}}}\left(\mathrm{t}_{\mathrm{i}}\right) \mathrm{dt}_{\mathrm{i}} ; \quad \mathrm{t}_{\mathrm{D}_{\mathrm{i}}}<\mathrm{T}_{\mathrm{i}} \leq \mathrm{T}_{\mathrm{Max}_{\mathrm{i}}} \\
& \mathrm{B}_{\mathrm{i}}=\beta_{\mathrm{i}} \int_{\mathrm{t}_{\mathrm{D}_{\mathrm{i}}}}^{\mathrm{T}_{\mathrm{MAx}_{\mathrm{i}}}}\left(\mathrm{D}_{\mathrm{i}} \mathrm{T}_{\mathrm{i}}-\mathrm{R}_{\mathrm{i}}\right) \mathrm{f}_{\mathrm{T}_{\mathrm{i}}}\left(\mathrm{t}_{\mathrm{i}}\right) \mathrm{dt}_{\mathrm{i}} ; \quad \mathrm{t}_{\mathrm{D}_{\mathrm{i}}}<\mathrm{T}_{\mathrm{i}}<\mathrm{T}_{\mathrm{Max}_{\mathrm{i}}} \\
& I_{i}=\int_{T_{\text {Min }_{i}}}^{t_{D_{i}}}\left(R_{i} T_{i}-\frac{D_{i} T_{i}^{2}}{2}\right) f_{T_{i}}\left(t_{i}\right) d t_{i}+\int_{t_{D_{i}}}^{T_{\text {Max }_{i}}}\left(\frac{R_{i}^{2}}{2 D_{i}}\right) f_{T_{i}}\left(t_{i}\right) d t_{i}
\end{aligned}
$$

Presenting the constraints: As the total available warehouse space is $\mathrm{F}$, the space required for each unit of the $i^{\text {th }}$ product is $f_{i}$ and the inventory level of the $i^{\text {th }}$ product is $R_{i}$, the space constraint will be:

$$
\mathrm{f}_{\mathrm{i}} \mathrm{R}_{\mathrm{i}} \leq \mathrm{F}
$$

Since the total available budget is TB, the cost for each unit of product is $\mathrm{W}$ and the order quantity is $\mathrm{Q}$, the budget constraint is:

$$
\mathrm{W}_{\mathrm{i}} \mathrm{Q}_{\mathrm{i}} \leq \mathrm{TB}
$$

Knowing that the shortages only occur when the cycle time is more than $t_{\mathrm{Di}}$ and that the lower limit for the service level is $\mathrm{SL}_{\mathrm{i}}$, then:

$$
P\left(T_{i}>t_{D_{i}}\right)=\int_{\frac{R_{i}}{D_{i}}}^{T_{\text {Max }_{i}}} f_{T_{i}}\left(t_{i}\right) d t_{i} \leq 1-S L_{i}
$$

In short, the complete mathematical model of the single product inventory problem with crisp demand and no discount is: 


$$
\begin{aligned}
& \operatorname{Max} Z_{i}=\left(P_{i}-W_{i}\right)\left[\int_{T_{M_{i n}}}^{\frac{R_{i}}{D_{i}}}\left(D_{i} T_{i}\right) f_{T_{i}}\left(t_{i}\right) d t_{i}+\int_{\frac{R_{i}}{D_{i}}}^{T_{M_{i}}}\left(R_{i}+\beta_{i}\left(D_{i} T_{i}-R_{i}\right)\right) f_{T_{i}}\left(t_{i}\right) d t_{i}\right] \\
& -h_{i}\left[\int_{T_{M n_{i}}}^{\frac{R_{i}}{D_{i}}}\left(R_{i} T_{i}-\frac{D_{i} T_{i}^{2}}{2}\right) f_{T_{i}}\left(t_{i}\right) d t_{i}+\int_{\frac{R_{i}}{D_{i}}}^{T_{M x_{i}}} \frac{R_{i}^{2}}{2 D_{i}} f_{T_{i}}\left(t_{i}\right) d t_{i}\right] \\
& -\pi_{i} \beta_{i}\left[\int_{\frac{R_{i}}{D_{i}}}^{T_{M a x_{i}}}\left(D_{i} T_{i}-R_{i}\right) f_{T_{i}}\left(t_{i}\right) d t_{i}\right]-\left(1-\beta_{i}\right) \hat{\pi}_{i}\left[\int_{\frac{R_{i}}{D_{i}}}^{T_{M_{i}}}\left(D_{i} T_{i}-R_{i}\right) f_{T_{i}}\left(t_{i}\right) d t_{i}\right] \\
& \text { s.t.: } \\
& \mathrm{f}_{\mathrm{i}} \mathrm{R}_{\mathrm{i}} \leq \mathrm{F} \\
& \mathrm{W}_{\mathrm{i}}\left[\int_{\mathrm{T}_{\mathrm{Min}_{\mathrm{i}}}}^{\mathrm{t}_{\mathrm{D}_{\mathrm{i}}}} \mathrm{D}_{\mathrm{i}} \mathrm{T}_{\mathrm{i}} \mathrm{f}_{\mathrm{T}_{\mathrm{i}}}\left(\mathrm{t}_{\mathrm{i}}\right) \mathrm{dt}_{\mathrm{i}}+\int_{\mathrm{t}_{\mathrm{D}_{\mathrm{i}}}}^{\mathrm{T}_{\text {Max }_{\mathrm{i}}}}\left(\mathrm{R}_{\mathrm{i}}+\beta_{\mathrm{i}}\left(\mathrm{D}_{\mathrm{i}} \mathrm{T}_{\mathrm{i}}-\mathrm{R}_{\mathrm{i}}\right)\right) \mathrm{f}_{\mathrm{T}_{\mathrm{i}}}\left(\mathrm{t}_{\mathrm{i}}\right) d \mathrm{t}_{\mathrm{i}}\right] \leq \mathrm{TB} \\
& P\left(T_{i}>t_{D_{i}}\right)=\int_{\frac{R_{i}}{D_{i}}}^{T_{\text {Max }_{i}}} f_{T_{i}}\left(t_{i}\right) d t_{i} \leq 1-S L_{i} \\
& \mathrm{R}_{\mathrm{i}} \geq 0 \text { and Integer }
\end{aligned}
$$

Single product model-back ordered and lost sales cases with discount: In this section, we assume that an incremental discount policy is applicable to purchase the product. In incremental discount policy, the purchasing cost for each unit of the $i^{\text {th }}$ product depends on its order quantity and is assumed to be:

$$
\mathrm{W}_{\mathrm{i}}= \begin{cases}\mathrm{W}_{\mathrm{i} 1} & 0<\mathrm{Q} \leq \mathrm{q}_{\mathrm{i} 1} \\ \mathrm{~W}_{\mathrm{i} 2} & \mathrm{q}_{\mathrm{i} 1}<\mathrm{Q} \leq \mathrm{q}_{\mathrm{i} 2} \\ \vdots & \vdots \\ \mathrm{W}_{\mathrm{iT}} & \mathrm{q}_{\mathrm{iT}} \leq \mathrm{Q}_{\mathrm{i}}\end{cases}
$$

The purchasing cost associated with this policy is calculated as follows:

$$
\mathrm{C}_{\mathrm{p}_{\mathrm{i}}}=\left\{\begin{array}{lc}
\mathrm{W}_{\mathrm{i} 1} \mathrm{Q}_{\mathrm{i}} & 0<\mathrm{Q}_{\mathrm{i}} \leq \mathrm{q}_{\mathrm{i} 1} \\
\mathrm{~W}_{\mathrm{i} 1} \mathrm{q}_{\mathrm{i} 1}+\mathrm{W}_{\mathrm{i} 2}\left(\mathrm{Q}_{\mathrm{i}}-\mathrm{q}_{\mathrm{i} 1}\right) & \mathrm{q}_{\mathrm{i} 1}<\mathrm{Q}_{\mathrm{i}} \leq \mathrm{q}_{\mathrm{i} 2} \\
\vdots & \vdots \\
\mathrm{W}_{\mathrm{i} 1} \mathrm{q}_{\mathrm{i} 1}+\mathrm{W}_{\mathrm{i} 2}\left(\mathrm{q}_{\mathrm{i} 2}-\mathrm{q}_{\mathrm{i} 1}\right)+\cdots+\mathrm{W}_{\mathrm{iT}}\left(\mathrm{Q}_{\mathrm{i}}-\mathrm{q}_{\mathrm{iT}-1}\right) & \mathrm{q}_{\mathrm{iT}} \leq \mathrm{Q}_{\mathrm{i}}
\end{array}\right.
$$

where, for $\mathrm{j}=1,2, \ldots, \mathrm{T}, \mathrm{q}_{\mathrm{ij}}$ and $\mathrm{W}_{\mathrm{ij}}$ are the discount points and the purchasing costs for each unit of the $\mathrm{i}^{\text {th }}$ product that corresponds to the $\mathrm{j}^{\text {th }}$ discount break point, respectively.

In order to include the discount policy in the inventory model, using Eq. 16, the purchasing cost will be modelled as: 


$$
\begin{gathered}
\mathrm{C}_{\mathrm{p}_{\mathrm{i}}}=\mathrm{W}_{\mathrm{i} 1} \mathrm{Q}_{\mathrm{i} 1}+\mathrm{W}_{\mathrm{i} 2} \mathrm{Q}_{\mathrm{i} 2}+\ldots+\mathrm{W}_{\mathrm{iT}} \mathrm{Q}_{\mathrm{iT}} \\
\mathrm{Q}_{\mathrm{i}}=\mathrm{Q}_{\mathrm{i} 1}+\mathrm{Q}_{\mathrm{i} 2}+\ldots+\mathrm{Q}_{\mathrm{iT}} \\
\mathrm{q}_{\mathrm{i} 1} \mathrm{Y}_{\mathrm{i} 2} \leq \mathrm{Q}_{\mathrm{i} 1} \leq \mathrm{q}_{\mathrm{i} 1} \mathrm{Y}_{\mathrm{i} 1} \\
\left(\mathrm{q}_{\mathrm{i} 2}-\mathrm{q}_{\mathrm{i} 1}\right) \mathrm{Y}_{\mathrm{i} 3} \leq \mathrm{Q}_{\mathrm{i} 2} \leq\left(\mathrm{q}_{\mathrm{i} 2}-\mathrm{q}_{\mathrm{i} 1}\right) \mathrm{Y}_{\mathrm{i} 2} \\
\vdots \\
0 \leq \mathrm{Q}_{\mathrm{iT}} \leq \mathrm{M} \mathrm{Y}_{\mathrm{iT}} \quad \mathrm{M} \text { is a large digit } \\
\mathrm{Y}_{\mathrm{i} 1} \geq \mathrm{Y}_{\mathrm{i} 2} \geq \ldots \geq \mathrm{Y}_{\mathrm{iT}} \\
\mathrm{Y}_{\mathrm{ij}}=0,1 ; \mathrm{j}=1,2, \ldots, \mathrm{T}
\end{gathered}
$$

By this modeling, the inventory model of the single product problem with incremental discount policy becomes:

$$
\begin{aligned}
& \operatorname{Max} \mathrm{Z}_{\mathrm{i}}=\mathrm{P}_{\mathrm{i}} \mathrm{Q}_{\mathrm{i}}-\mathrm{h}_{\mathrm{i}}^{\prime \prime} \mathrm{I}_{\mathrm{i}}-\pi_{\mathrm{i}} \mathrm{B}_{\mathrm{i}}-\hat{\pi}_{\mathrm{i}} \mathrm{L}_{\mathrm{i}}-\sum_{\mathrm{j}=1}^{\mathrm{T}} \mathrm{W}_{\mathrm{ij}} \mathrm{Q}_{\mathrm{ij}} \\
& P\left[\begin{array}{l}
\int_{T_{\mathrm{Min}_{\mathrm{i}}}}^{\frac{\mathrm{R}_{\mathrm{i}}}{\mathrm{D}_{\mathrm{i}}}}\left(\mathrm{D}_{\mathrm{i}} \mathrm{T}_{\mathrm{i}}\right) \mathrm{f}_{\mathrm{T}_{\mathrm{i}}}\left(\mathrm{t}_{\mathrm{i}}\right) d \mathrm{t}_{\mathrm{i}} \\
+\int_{\frac{\mathrm{R}_{\mathrm{i}}}{\mathrm{D}_{\mathrm{i}}}}^{\mathrm{T}_{\mathrm{Maz}_{\mathrm{i}}}}\left(\mathrm{R}_{\mathrm{i}}+\beta_{\mathrm{i}}\left(\mathrm{D}_{\mathrm{i}} \mathrm{T}_{\mathrm{i}}-\mathrm{R}_{\mathrm{i}}\right)\right) \mathrm{f}_{\mathrm{T}_{\mathrm{i}}}\left(\mathrm{t}_{\mathrm{i}}\right) \mathrm{dt}_{\mathrm{i}}
\end{array}\right] \\
& -h_{i}^{\prime \prime}\left[\begin{array}{l}
\int_{T_{\text {Mini }_{i}}}^{\frac{R_{i}}{D_{i}}}\left(R_{i} T_{i}-\frac{D_{i} T_{i}^{2}}{2}\right) f_{T_{i}}\left(t_{i}\right) d t_{i} \\
+\int_{\frac{R_{i}}{D_{i}}}^{T_{M_{i}}} \frac{R_{i}^{2}}{2 D_{i}} f_{T_{i}}\left(t_{i}\right) d t_{i}
\end{array}\right] \\
& -\pi_{i} \beta_{i}\left[\int_{\frac{R_{i}}{D_{i}}}^{T_{\operatorname{Max}_{i}}}\left(D_{i} T_{i}-R_{i}\right) f_{T_{i}}\left(t_{i}\right) d t_{i}\right] \\
& -\hat{\pi}_{\mathrm{i}}\left(1-\beta_{\mathrm{i}}\right)\left[\int_{\frac{\mathrm{R}_{\mathrm{i}}}{\mathrm{D}_{\mathrm{i}}}}^{\mathrm{T}_{\mathrm{Max}_{\mathrm{i}}}}\left(\mathrm{D}_{\mathrm{i}} \mathrm{T}_{\mathrm{i}}-\mathrm{R}_{\mathrm{i}}\right) \mathrm{f}_{\mathrm{T}_{\mathrm{i}}}\left(\mathrm{t}_{\mathrm{i}}\right) \mathrm{dt} \mathrm{t}_{\mathrm{i}}-\sum_{\mathrm{j}=1}^{\mathrm{T}} \mathrm{W}_{\mathrm{ij}} \mathrm{Q}_{\mathrm{ij}}\right.
\end{aligned}
$$

s.t.:

$$
\begin{aligned}
& \mathrm{f}_{\mathrm{i}} \mathrm{R}_{\mathrm{i}} \leq \mathrm{F} \\
& \sum_{\mathrm{j}=1}^{\mathrm{T}} \mathrm{W}_{\mathrm{ij}} \mathrm{Q}_{\mathrm{ij}} \leq \mathrm{TB} \\
& \int_{\frac{\mathrm{R}_{\mathrm{i}}}{\mathrm{D}_{\mathrm{i}}}}^{\mathrm{T}_{\mathrm{Max}_{\mathrm{i}}}} \mathrm{f}_{\mathrm{T}_{\mathrm{i}}}\left(\mathrm{t}_{\mathrm{i}}\right) \mathrm{dt}_{\mathrm{i}} \leq 1-\mathrm{SL}_{\mathrm{i}} \\
& \mathrm{Q}_{\mathrm{i}}=\mathrm{Q}_{\mathrm{i} 1}+\mathrm{Q}_{\mathrm{i} 2}+\ldots+\mathrm{Q}_{\mathrm{iT}} \\
& \mathrm{q}_{\mathrm{i} 1} \mathrm{Y}_{\mathrm{i} 2} \leq \mathrm{Q}_{\mathrm{i} 1} \leq \mathrm{q}_{\mathrm{i} 1} \mathrm{Y}_{\mathrm{i} 1} \\
& \left(\mathrm{q}_{\mathrm{i} 2}-\mathrm{q}_{\mathrm{i} 1}\right) \mathrm{Y}_{\mathrm{i} 3} \leq \mathrm{Q}_{\mathrm{i} 2} \leq\left(\mathrm{q}_{\mathrm{i} 2}-\mathrm{q}_{\mathrm{i} 1}\right) \mathrm{Y}_{\mathrm{i} 2} \\
& \quad \quad \quad \\
& \quad 0 \leq \mathrm{Q}_{\mathrm{iT}} \leq \mathrm{MY} \mathrm{Y}_{\mathrm{iT}} \\
& \mathrm{Y}_{\mathrm{i} 1} \geq \mathrm{Y}_{\mathrm{i} 2} \geq \cdots \geq \mathrm{Y}_{\mathrm{iT}} \\
& \mathrm{Y}_{\mathrm{ij}}=0,1 ; \mathrm{j}=1,2, \ldots, \mathrm{T} \text { a large digit } \\
& \mathrm{R}_{\mathrm{i}} \geq 0 \text { and Integer }
\end{aligned}
$$

Single-product model with discount, fuzzy purchasing and holding costs: The single-product inventory model with crisp purchasing and holding cost and incremental discount of (18) can be easily extended to single product models with fuzzy purchasing and holding cost as follows:

$\operatorname{Max} Z\left(R_{i}, \tilde{W}_{i}, \widetilde{h_{i}^{\prime}}\right)=P_{i} Q_{i}-\widetilde{h_{i}^{\prime}} I_{i}-\pi_{i} B_{i}-\hat{\pi}_{i} L_{i}-\sum_{j=1}^{T} \tilde{W}_{i j} Q_{i j}$

$$
\begin{aligned}
& =P_{i}\left[\begin{array}{l}
\int_{T_{M_{i n}}}^{\frac{R_{i}}{D_{i}}}\left(D_{i} T_{i}\right) f_{T_{i}}\left(t_{i}\right) d t_{i}+ \\
\int_{\frac{R_{i}}{D_{i}}}^{T_{M a x_{i}}}\left(R_{i}+\beta_{i}\left(D_{i} T_{i}-R_{i}\right)\right) f_{T_{i}}\left(t_{i}\right) d t_{i}
\end{array}\right] \\
& -\tilde{h}_{i}^{\prime \prime}\left[\begin{array}{l}
\int_{T_{M_{i}}}^{\frac{R_{i}}{D_{i}}}\left(R_{i} T_{i}-\frac{D_{i} T_{i}^{2}}{2}\right) f_{T_{i}}\left(t_{i}\right) d t_{i} \\
+\int_{\frac{R_{i}}{D_{i}}}^{T_{M_{i}}} \frac{R_{i}^{2}}{2 D_{i}} f_{T_{i}}\left(t_{i}\right) d t_{i}
\end{array}\right] \\
& -\pi_{i} \beta_{i}\left[\int_{\frac{R_{i}}{D_{i}}}^{T_{M_{i}}}\left(D_{i} T_{i}-R_{i}\right) f_{T_{i}}\left(t_{i}\right) d t_{i}\right] \\
& -\hat{\pi}_{i}\left(1-\beta_{i}\right)\left[\int_{\frac{R_{i}}{D_{i}}}^{T_{M x_{i}}}\left(D_{i} T_{i}-R_{i}\right) f_{T_{i}}\left(t_{i}\right) d t_{i}-\sum_{j=1}^{T} \tilde{W}_{i j} Q_{i j}\right.
\end{aligned}
$$

s.t:

$$
\begin{aligned}
& \mathrm{f}_{\mathrm{i}} \mathrm{R}_{\mathrm{i}} \leq \mathrm{F} \\
& \sum_{\mathrm{j}=1}^{\mathrm{T}} \tilde{\mathrm{W}}_{\mathrm{ij}} \mathrm{Q}_{\mathrm{ij}} \leq \mathrm{TB} \\
& \int_{\frac{\mathrm{R}_{\mathrm{i}}}{\mathrm{D}_{\mathrm{i}}}}^{\mathrm{T}_{\mathrm{Max}_{\mathrm{i}}}} \mathrm{f}_{\mathrm{T}_{\mathrm{i}}}\left(\mathrm{t}_{\mathrm{i}}\right) \mathrm{dt}_{\mathrm{i}} \leq 1-\mathrm{SL}_{\mathrm{i}} \\
& \mathrm{Q}_{\mathrm{i}}=\mathrm{Q}_{\mathrm{i} 1}+\mathrm{Q}_{\mathrm{i} 2}+\ldots+\mathrm{Q}_{\mathrm{iT}} \\
& \mathrm{q}_{\mathrm{i} 1} \mathrm{Y}_{\mathrm{i} 2} \leq \mathrm{Q}_{\mathrm{i} 1} \leq \mathrm{q}_{\mathrm{i} 1} \mathrm{Y}_{\mathrm{i} 1} \\
& \left(\mathrm{q}_{\mathrm{i} 2}-\mathrm{q}_{\mathrm{i} 1}\right) \mathrm{Y}_{\mathrm{i} 3} \leq \mathrm{Q}_{\mathrm{i} 2} \leq\left(\mathrm{q}_{\mathrm{i} 2}-\mathrm{q}_{\mathrm{i} 1}\right) \mathrm{Y}_{\mathrm{i} 2} \\
& \quad \quad \quad \mathrm{M} \text { is a large digit } \\
& 0 \leq \mathrm{Q}_{\mathrm{iT}} \leq \mathrm{MY} \mathrm{Y}_{\mathrm{iT}} \\
& \mathrm{Y}_{\mathrm{i} 1} \geq \mathrm{Y}_{\mathrm{i} 2} \geq \cdots \geq \mathrm{Y}_{\mathrm{iT}} \\
& \mathrm{Y}_{\mathrm{ij}}=0,1 \\
& \mathrm{R}_{\mathrm{i}} \geq 0, \text { Integer }
\end{aligned}
$$

In the next section, we extend the models in (19) to multi-product models.

Multi-product models: The single-product inventory models of (19) can be easily extended to a multiple 
product. In these models, we consider two probability density functions for $\mathrm{T}_{\mathrm{i}}$ as follow:

$\mathbf{T}_{\mathbf{i}}$ follows a uniform distribution: In this case the probability density function of $\mathrm{T}_{\mathrm{i}}$ is $\mathrm{f}_{\mathrm{T}_{\mathrm{i}}}\left(\mathrm{t}_{\mathrm{i}}\right)=\frac{1}{\mathrm{~T}_{\max _{\mathrm{i}}}-\mathrm{T}_{\min _{\mathrm{i}}}}$. Accordingly, (19) will change to (20) as:

$$
\begin{aligned}
& \operatorname{Max} Z\left(\mathrm{R}_{\mathrm{i}}, \tilde{\mathrm{W}}_{\mathrm{i}}, \widetilde{\mathrm{h}_{\mathrm{i}}^{\prime}}\right)=\sum_{\mathrm{i}=1}^{\mathrm{n}}\left[\frac{\widetilde{\mathrm{h}_{\mathrm{i}}^{\prime}}}{6 \mathrm{D}_{\mathrm{i}}^{2}\left(\mathrm{~T}_{\mathrm{Max}_{\mathrm{i}}}-\mathrm{T}_{\mathrm{Min}_{\mathrm{i}}}\right)}\right] \mathrm{R}_{\mathrm{i}}^{3} \\
& -\sum_{\mathrm{i}=1}^{\mathrm{n}}\left[\frac{\left(1-\beta_{\mathrm{i}}\right) \mathrm{p}_{\mathrm{i}}+\widetilde{\mathrm{h}_{\mathrm{i}}^{\prime}} \mathrm{T}_{\mathrm{Max}_{\mathrm{i}}}+\pi_{\mathrm{i}} \beta_{\mathrm{i}}+\hat{\pi}_{\mathrm{i}}\left(1-\beta_{\mathrm{i}}\right)}{2 \mathrm{D}_{\mathrm{i}}\left(\mathrm{T}_{\operatorname{Max}_{\mathrm{i}}}-\mathrm{T}_{\operatorname{Min}_{\mathrm{i}}}\right)}\right] \mathrm{R}_{\mathrm{i}}^{2}
\end{aligned}
$$

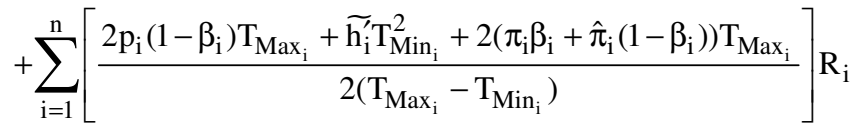

$$
\begin{aligned}
& +\sum_{i=1}^{n}\left[\frac{3 p_{i} D_{i}\left(\beta_{i} T_{\operatorname{Max}_{i}}^{2}-T_{\operatorname{Min}_{i}}^{2}\right)-\tilde{h}_{i}^{\prime \prime} T_{\operatorname{Min}_{i}}^{3} D_{i}-3\left(\pi_{i} \beta_{i}+\hat{\pi}_{i}\left(1-\beta_{i}\right)\right) T_{M_{i}}^{2} D_{i}}{6\left(T_{\operatorname{Max}_{i}}-T_{M_{i n}}\right)}\right]-\sum_{i=1}^{n} \sum_{j=1}^{T} \tilde{W}_{i j} Q_{i j}
\end{aligned}
$$

s.t.:

$$
\begin{aligned}
& \sum_{i=1}^{n} \sum_{j=1}^{T} \tilde{\mathrm{W}}_{\mathrm{ij}} \mathrm{Q}_{\mathrm{ij}} \leq \mathrm{TB} \\
& \sum_{i=1}^{n} f_{i} R_{i} \leq F \\
& \frac{\mathrm{D}_{\mathrm{i}} \mathrm{T}_{\mathrm{Max}_{\mathrm{i}}}-\mathrm{R}_{\mathrm{i}}}{\mathrm{D}_{\mathrm{i}}\left(\mathrm{T}_{\operatorname{Max}_{\mathrm{i}}}-\mathrm{T}_{\operatorname{Min}_{\mathrm{i}}}\right)} \leq 1-\mathrm{SL}_{\mathrm{i}} \quad \forall \mathrm{i}: \mathrm{i}=1,2, \ldots, \mathrm{n} \\
& \frac{\left(\beta_{\mathrm{i}}-1\right) \mathrm{R}_{\mathrm{i}}^{2}+\left(2 \mathrm{D}_{\mathrm{i}} \mathrm{T}_{\mathrm{Max}_{\mathrm{i}}}\left(1-\beta_{\mathrm{i}}\right)\right) \mathrm{R}_{\mathrm{i}}+\left(\beta_{\mathrm{i}} \mathrm{T}_{\mathrm{Max}_{\mathrm{i}}}^{2}-\mathrm{T}_{\mathrm{Min}_{\mathrm{i}}}^{2}\right) \mathrm{D}_{\mathrm{i}}^{2}}{2 \mathrm{D}_{\mathrm{i}}\left(\mathrm{T}_{\mathrm{Max}_{\mathrm{i}}}-\mathrm{T}_{\mathrm{Min}_{\mathrm{i}}}\right)} \\
& =\sum_{\mathrm{j}=1}^{\mathrm{T}} \mathrm{Q}_{\mathrm{ij}} \quad \forall \mathrm{i}, \quad \mathrm{i}=1,2, \ldots, \mathrm{n} \\
& \mathrm{q}_{1 \mathrm{j}} \mathrm{Y}_{2 \mathrm{j}} \leq \mathrm{Q}_{1 \mathrm{j}} \leq \mathrm{q}_{1 \mathrm{j}} \mathrm{Y}_{1 \mathrm{j}} \quad \forall \mathrm{j}, \quad \mathrm{j}=1,2, \ldots, \mathrm{T} \\
& \left(\mathrm{q}_{\mathrm{ij}}-\mathrm{q}_{\mathrm{i}-1, \mathrm{j}}\right) \mathrm{Y}_{\mathrm{ij}} \leq \mathrm{Q}_{\mathrm{ij}} \leq\left(\mathrm{q}_{\mathrm{ij}}-\mathrm{q}_{\mathrm{i}-1, \mathrm{j}}\right) \mathrm{Y}_{\mathrm{i}-1, \mathrm{j}} \quad \forall \mathrm{i}, \mathrm{i}=2, \ldots, \mathrm{n}_{\mathrm{j}}-1 \quad \forall \mathrm{j}, \quad \mathrm{j}=1,2, \ldots, \mathrm{T} \\
& 0 \leq \mathrm{Q}_{\mathrm{n}_{\mathrm{j}} \mathrm{j}} \leq \mathrm{MY}_{\mathrm{n}_{\mathrm{j}} \mathrm{j}} \quad \forall \mathrm{j}, \quad \mathrm{j}=1,2, \ldots, \mathrm{T}, \quad \mathrm{M} \text { is a large digit } \\
& Y_{1 j} \geq Y_{2 j} \geq \cdots \geq Y_{n_{j} j} \quad \forall j, \quad j=1,2, \cdots, T \\
& \mathrm{Y}_{\mathrm{ij}}=0,1 \quad \forall \mathrm{j}, \quad \mathrm{j}=1,2, \cdots \mathrm{T}, \quad \forall \mathrm{i}, \quad \mathrm{i}=1,2, \cdots, \mathrm{n} \\
& \mathrm{R}_{\mathrm{i}} \geq 0, \text { Integer } \quad \forall \mathrm{i}: \mathrm{i}=1,2, \ldots, \mathrm{n}
\end{aligned}
$$

$\mathrm{T}_{\mathrm{I}}$ follows an exponential distribution: If $\mathrm{T}_{\mathrm{i}}$ follows an exponential distribution with parameter $\lambda_{\mathrm{i}}$, then the probability density function of $\mathrm{T}_{\mathrm{i}}$ will be $\mathrm{f}_{\mathrm{T}_{\mathrm{i}}}\left(\mathrm{t}_{\mathrm{i}}\right)=\lambda_{\mathrm{i}} \mathrm{e}^{-\lambda_{\mathrm{i}} \mathrm{T}_{\mathrm{i}}}$. In this case, the model is shown in (21) as: 


$$
\begin{aligned}
& \operatorname{Max}: \mathrm{Z}\left(\mathrm{R}_{\mathrm{i}}, \tilde{\mathrm{W}}_{\mathrm{i}}, \widetilde{\mathrm{h}_{\mathrm{i}}^{\prime}}\right)=\sum_{\mathrm{i}=1}^{\mathrm{n}}-\frac{\left[\left(\mathrm{p}_{\mathrm{i}}+\hat{\pi}_{\mathrm{i}}\right)\left(1-\beta_{\mathrm{i}}\right)+\pi_{\mathrm{i}} \beta_{\mathrm{i}}\right]}{\lambda_{\mathrm{i}}} \mathrm{D}_{\mathrm{i}} \mathrm{e}^{-\left(\frac{\lambda_{\mathrm{i}}}{\mathrm{D}_{\mathrm{i}}}\right) \mathrm{R}_{\mathrm{i}}} \\
& +\sum_{i=1}^{n} \frac{\widetilde{\mathrm{h}_{i}^{\prime}} \mathrm{D}_{\mathrm{i}}}{\lambda_{\mathrm{i}}{ }^{2}}\left(1-\mathrm{e}^{-\left(\frac{\lambda_{\mathrm{i}}}{\mathrm{D}_{\mathrm{i}}}\right) \mathrm{R}_{\mathrm{i}}}\right)+\sum_{\mathrm{i}=1}^{\mathrm{n}} \frac{\mathrm{p}_{\mathrm{i}} \mathrm{D}_{\mathrm{i}}-\widetilde{\mathrm{h}_{\mathrm{i}}^{\prime}} \mathrm{R}_{\mathrm{i}}}{\lambda_{\mathrm{i}}}-\sum_{\mathrm{i}=1}^{\mathrm{n}} \sum_{\mathrm{j}=1}^{\mathrm{T}} \tilde{\mathrm{W}}_{\mathrm{ij}} \mathrm{Q}_{\mathrm{ij}} \\
& \text { s.t.: }
\end{aligned}
$$

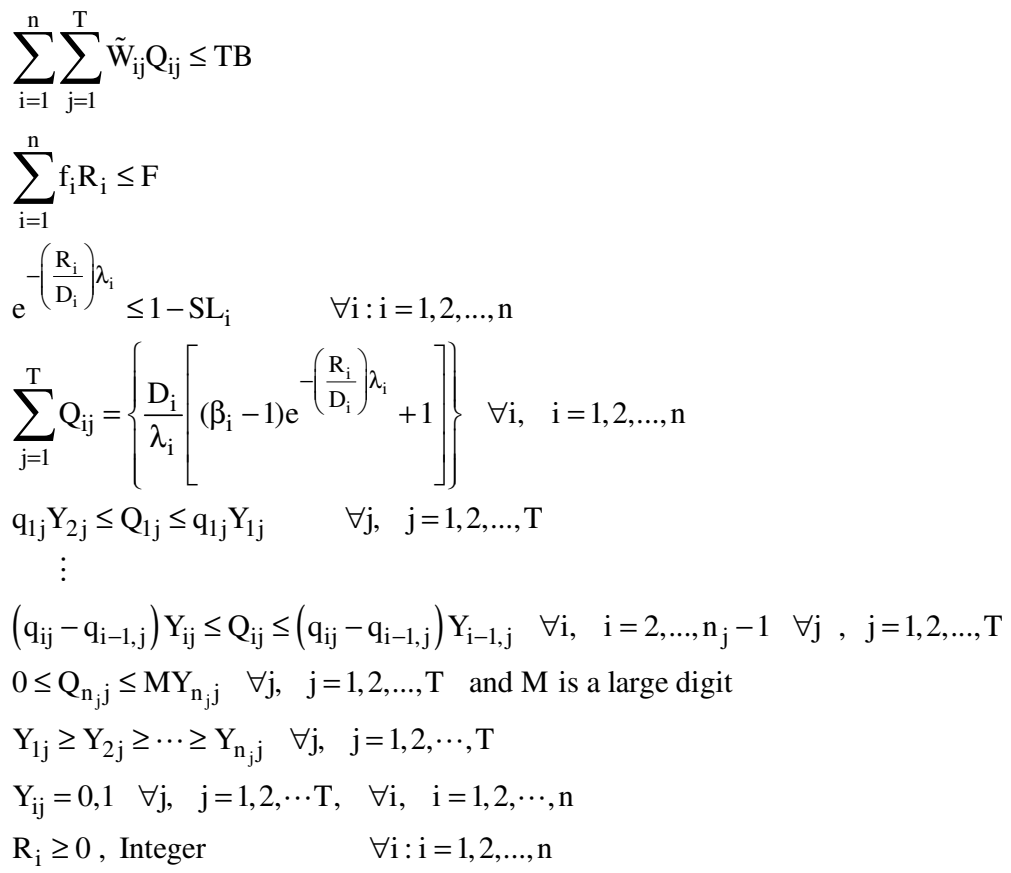

In the next section, we will introduce a hybrid intelligent algorithm to solve the model.

\section{A HYBRID INTELLIGENT ALGORITHM}

Since the models in (20) and (21) are fuzzy mixed integer-nonlinear in nature, reaching an analytical solution (if any) to the problem is difficult ${ }^{[21]}$. In order to solve the model under different criteria, we develop a hybrid intelligent algorithm of fuzzy simulation and genetic algorithm.

Fuzzy simulation: In order to estimate the uncertain purchasing price and holding cost of the fuzzy model, since the holding cost is a function of its corresponding purchasing cost, an estimate of the former cost will provide an estimate of the latter cost. As a result, in the simulation technique used for the estimation, denoting $\tilde{\mathrm{W}}_{\mathrm{ij}}$ by $\tilde{\mathrm{W}}_{\mathrm{ij}}=\left(\tilde{\mathrm{W}}_{1 \mathrm{j}}, \tilde{\mathrm{W}}_{2 \mathrm{j}}, \ldots, \tilde{\mathrm{W}}_{\mathrm{nj}}\right), \mu$ as the membership function of $\tilde{\mathrm{W}}$ and $\mu_{\mathrm{ij}}$ are the membership functions of $\tilde{\mathrm{W}}_{\mathrm{ij}}$, we randomly generate $\mathrm{W}_{\mathrm{ijk}}$ from the $\alpha$-level sets of fuzzy variables $\tilde{\mathrm{W}}_{\mathrm{ij}}, \mathrm{i}=1,2, \ldots, \mathrm{n}, \mathrm{j}=1,2, \ldots, \mathrm{T}$ and $\mathrm{k}=1,2, \ldots, \mathrm{K} \quad$ as $\quad \mathrm{W}_{\mathrm{jk}}=\left(\mathrm{W}_{1 \mathrm{jk}}, \mathrm{W}_{2 \mathrm{jk}}, \cdots, \mathrm{W}_{\mathrm{njk}}\right)$ and $\mu\left(\mathrm{W}_{\mathrm{jk}}\right)=\mu_{1}\left(\mathrm{~W}_{1 \mathrm{jk}}\right) \wedge \mu_{2}\left(\mathrm{~W}_{2 \mathrm{jk}}\right) \wedge, \cdots, \wedge \mu_{\mathrm{n}}\left(\mathrm{W}_{\mathrm{njk}}\right)$, where $\alpha$ is a sufficiently small positive number.

Based on the definition in Eq. 11, the expected value of the fuzzy variable is:

$$
\begin{aligned}
\mathrm{E}[\mathrm{Z}(\mathrm{R}, \tilde{\mathrm{W}}, \tilde{\mathrm{h}})]= & \int_{0}^{+\infty} \operatorname{Cr}\{\mathrm{Z}(\mathrm{R}, \tilde{\mathrm{W}}, \tilde{\mathrm{h}}) \geq \mathrm{r}\} \mathrm{dr} \\
& -\int_{-\infty}^{0} \operatorname{Cr}\{\mathrm{Z}(\mathrm{R}, \tilde{\mathrm{W}}, \tilde{\mathrm{h}}) \leq \mathrm{r}\} \mathrm{dr}
\end{aligned}
$$

Then, provided $\mathrm{O}$ is sufficiently large, for any number $r \geq 0, \operatorname{Cr}\{Z(R, \tilde{W}, \tilde{h}) \geq r\}$ can be estimated by:

$\operatorname{Cr}\{\mathrm{Z}(\mathrm{R}, \tilde{\mathrm{W}}, \tilde{\mathrm{h}}) \geq \mathrm{r}\}=\frac{1}{2}\left(\begin{array}{c}\operatorname{Max}_{\mathrm{k}=1,2, \ldots, \mathrm{O}}\left\{\mu_{\mathrm{k}} \mid \mathrm{Z}(\mathrm{R}, \tilde{\mathrm{W}}, \tilde{\mathrm{h}}) \geq \mathrm{r}\right\} \\ +1-\underset{\mathrm{k}=1,2, \ldots, \mathrm{O}}{\operatorname{Max}}\left\{\mu_{\mathrm{k}} \mid \mathrm{Z}(\mathrm{R}, \tilde{\mathrm{W}}, \tilde{\mathrm{h}})<\mathrm{r}\right\}\end{array}\right)$ 
and for any number $\mathrm{r}<0, \operatorname{Cr}\{\mathrm{Z}(\mathrm{R}, \tilde{\mathrm{W}}, \tilde{\mathrm{h}}) \leq \mathrm{r}\} \quad$ can be estimated by:

$$
\operatorname{Cr}\{\mathrm{Z}(\mathrm{R}, \tilde{\mathrm{W}}, \tilde{\mathrm{h}}) \leq \mathrm{r}\}=\frac{1}{2}\left(\begin{array}{l}
\operatorname{Max}_{\mathrm{k}=1,2, \ldots, \mathrm{O}}\left\{\mu_{\mathrm{k}} \mid \mathrm{Z}(\mathrm{R}, \tilde{\mathrm{W}}, \tilde{\mathrm{h}}) \leq \mathrm{r}\right\} \\
+1-\operatorname{Max}_{\mathrm{k}=1,2, \ldots, \mathrm{O}}\left\{\mu_{\mathrm{k}} \mid \mathrm{Z}(\mathrm{R}, \tilde{\mathrm{W}}, \tilde{\mathrm{h}})>\mathrm{r}\right\}
\end{array}\right)
$$

However, the procedure of estimating $\mathrm{Z}(\mathrm{R}, \tilde{\mathrm{W}}, \tilde{\mathrm{h}})$ in (23) and (24) is shown in algorithm (1).

- $\quad$ Set $\mathrm{E}=0$

- Randomly generate $\mathrm{W}_{\mathrm{ijk}}$ from $\alpha$-level sets of fuzzy variables $\tilde{W}_{\mathrm{ij}}$ and set $\mathrm{W}_{\mathrm{jk}}=\left(\mathrm{W}_{1 \mathrm{jk}}, \mathrm{W}_{2 \mathrm{jk}}, \cdots, \mathrm{W}_{\mathrm{njk}}\right)$ Set $a=Z\left(R, \tilde{W}_{j 1}, \tilde{h}\right) \wedge Z\left(R, \tilde{W}_{j 2}, \tilde{h}\right) \wedge \cdots \wedge Z\left(R, \tilde{W}_{j O}, \tilde{h}\right)$, $\mathrm{b}=\mathrm{Z}\left(\mathrm{R}, \tilde{\mathrm{W}}_{\mathrm{j} 1}, \tilde{\mathrm{h}}\right) \vee \mathrm{Z}\left(\mathrm{R}, \tilde{\mathrm{W}}_{\mathrm{j} 2}, \tilde{\mathrm{h}}\right) \vee \cdots \vee \mathrm{Z}\left(\mathrm{R}, \tilde{\mathrm{W}}_{\mathrm{jO}}, \tilde{\mathrm{h}}\right)$

- Randomly generate $\mathrm{r}$ from Uniform [a,b]

- If $\mathrm{r} \geq 0$, then $\mathrm{E} \leftarrow \mathrm{E}+\mathrm{Cr}\{\mathrm{Z}(\mathrm{R}, \tilde{\mathrm{W}}, \tilde{\mathrm{h}}) \geq \mathrm{r}\}$, otherwise, $\mathrm{E} \leftarrow \mathrm{E}-\mathrm{Cr}\{\mathrm{Z}(\mathrm{R}, \tilde{\mathrm{W}}, \tilde{\mathrm{h}}) \leq \mathrm{r}\}$

- $\quad$ Repeat 4 and 5 for $\mathrm{O}$ times

- Calculate $\mathrm{E}(\mathrm{Z}(\mathrm{R}, \tilde{\mathrm{W}}, \tilde{\mathrm{h}}))=\mathrm{a} \vee 0+\mathrm{b} \wedge 0+\mathrm{E} * \frac{\mathrm{b}-\mathrm{a}}{\mathrm{O}}$

Algorithm (1): Estimating Z(R, $\tilde{W}, \tilde{h})$.

Genetic algorithm: The main information unit of any living organism is the gene, which is a part of a chromosome that determines specific characteristics such as eye-color, complexion, hair-color, etc. The fundamental principal of Genetic Algorithms (GA) first was introduced by Holland ${ }^{[22]}$. Since then many researchers have applied and expanded this concept in different fields of study. Genetic algorithm was inspired by the concept of survival of the fittest. In genetic algorithms, the optimal solution is the winner of the genetic game and any potential solution is assumed to be a creature that is determined by different parameters. These parameters are considered as genes of chromosomes that could be assumed to be binary strings. In this algorithm, the better chromosome is the one that is nearer to the optimal solution. In applied applications of genetic algorithms, populations of chromosomes are created randomly. The number of these populations is different in each problem. Some hints about choosing the proper number of population exist in different reports by Man et al. ${ }^{[23]}$.

Genetic algorithms imitate the evolutionary process of species that reproduce. They therefore do not operate on a single current solution, but on a set of current solutions called population. New candidates for the solution are generated with a mechanism called crossover that combines part of the genetic patrimony of each parent and then applies a random mutation. If the new individual, called child or offspring, inherits good characteristics from his parents the probability of its survival increases. This process will continue until a stopping criterion is satisfied. Then, the best offspring is chosen as a near optimum solution.

In this research, the chromosomes are strings of the inventory levels of the products $\left(\mathrm{R}_{\mathrm{i}}\right)$. Each population or generation of chromosomes has the same size which is well-known as the population size and is denoted by $\mathrm{N}$. If $\mathrm{N}$ is relatively small, then a small search space will be investigated and the GA algorithm will be very slow. In this research, 10, 100 and 500 are chosen as different population sizes. In a crossover operation, it is necessary to mate pairs of chromosomes to create offspring. There are three types of crossover operations: single-point, multi-point and uniform ${ }^{[21]}$. In this research, we employ the single-point crossover that is applied to parent chromosomes with the possibility of $\mathrm{P}_{\mathrm{c}}=0.8,0.85$ and 0.9 . Mutation is the second operation in a GA method for exploring new solutions and it operates on each of the chromosomes resulted from the crossover operation. In mutation, we replace a gene with a randomly selected number within the boundaries of the parameter ${ }^{[21]}$. We create a random number $\mathrm{RN}$ between $(0,1)$ for each gene. If $\mathrm{RN}$ is less than a predetermined mutation probability $\mathrm{P}_{\mathrm{m}}$, then the mutation occur in the gene. Otherwise, the mutation operation is not performed in that gene. More precisely, assume that for a specific gene such as $a_{j}$ in $a$ chromosome $R_{j}$ the generated random number is less than $\mathrm{P}_{\mathrm{m}}$ and hence the gene is selected for mutation. Then, we change the value of $a_{j}$ to the new value $a_{j}^{*}$ according to Eq. 25 and 26, randomly and with the same probability:

$$
\begin{aligned}
& a_{j}^{*}=a_{j}+\left(u_{j}-a_{j}\right) \times r \times\left(1-\frac{i}{\max g e n}\right) \\
& a_{j}^{*}=a_{j}-\left(a_{j}-1_{j}\right) \times r \times\left(1-\frac{i}{\text { max gen }}\right)
\end{aligned}
$$

where, $l_{j}$ and $u_{j}$ are the lower and upper limits of the specified gene, $r$ is a uniform random variable between 0 and $1, i$ is the number of current generation and max gen is the maximum number of generations. Note that the value of $\mathrm{a}_{\mathrm{j}}$ is transferred to its right or left randomly by Eq. 25 and 26 respectively and $r$ is this percentage. 
Furthermore, $1-\frac{\mathrm{i}}{\max \text { gen }}$ is an index with a value close to one in the first generation and close to zero in the last generation that makes large mutations in the early generations and almost no mutation in the last generations. In this research, 0.076, 0.098 and 0.1 are employed as different values of the $\mathrm{P}_{\mathrm{m}}$ parameter. Furthermore, Algorithm (1) of section 5.1 is used to evaluate the objective function of this research.

The last step in a GA method is to check if the algorithm has found a solution that is good enough to meet the user's expectations. Stopping criteria is a set of conditions such that when satisfied a good solution is obtained. Different criteria used in literature are as follows: (1) Stopping of the algorithm after a specific number of generations, (2) no improvement in the objective function and (3) Reaching a specific value of the objective function. In this research, we stop when a predetermined number of consecutive generations is reached. The number of sequential generations depends on the specified problem and the expectations of the user.

In short, the steps involved in the hybrid method of fuzzy simulation and GA algorithm used in this research are:

- $\quad$ Setting the parameters $\mathrm{P}_{\mathrm{c}}, \mathrm{P}_{\mathrm{m}}$ and $\mathrm{N}$

- Initializing the population randomly

- Evaluating the objective function for all chromosomes based on Algorithm (1)

- $\quad$ Selecting individual for mating pool

- Applying the crossover operation for each pair of chromosomes with probability $\mathrm{P}_{\mathrm{c}}$
- Applying mutation operation for each chromosome with probability $\mathrm{P}_{\mathrm{m}}$

- $\quad$ Replacing the current population by the resulting mating pool

- Evaluating the objective function

- If stopping criteria is met, then stop. Otherwise, go to step 5

In order to demonstrate the proposed Hybrid intelligent algorithm and evaluate its performance, in the next section we bring a numerical example used in Ertogal and Rahim ${ }^{[20]}$. In this example, two cases of the uniform and the exponential distributions for the timeperiod between two replenishments are investigated.

\section{NUMERICAL EXAMPLES}

Consider a multi-product inventory control problem with eight products and general data given in Table 1. Table 2 shows the parameters of both the exponential and uniform distributions used for the timeperiod between two replenishments. The total available warehouse space and total budget are $\mathrm{F}=22000$ and $\mathrm{TB}=550000$, respectively. Table 3 shows the best combination and different values of the GA parameters used to obtain the solution. In this research, all the possible combinations of the GA parameters $\left(\mathrm{P}_{\mathrm{c}}, \mathrm{P}_{\mathrm{m}}\right.$ and $\mathrm{N})$ are employed and using the $\max (\max )$ criterion the best combination of the parameters has been selected. Table 4 shows the best result for the uniform and exponential distributions. Furthermore, the convergence paths of the best result of the objective function values in different generations of the uniform and the exponential distributions are shown in Fig. 4 and 5, respectively.

Table 1: General data

\begin{tabular}{lllllllll}
\hline Product & 1 & 2 & 3 & 4 & 5 & 6 & 7 & 8 \\
\hline $\mathrm{FI}_{\mathrm{i}}$ & 0.05 & 0.05 & 0.05 & 0.05 & 0.03 & 0.03 & 0.03 & 0.03 \\
$\pi_{\mathrm{i}}$ & 5 & 5 & 5 & 5 & 5 & 5 & 5 & 10 \\
$\hat{\pi}_{\mathrm{i}}$ & 10 & 10 & 10 & 10 & 10 & 10 & 03 & 10 \\
$\beta_{\mathrm{i}}$ & 0.5 & 0.9 & 0.9 & 0.5 & 0.5 & 0.9 & 0.9 & 0.5 \\
$\mathrm{f}_{\mathrm{i}}$ & 3 & 3 & 3 & 3 & 6 & 6 & 6 \\
$\tilde{\mathrm{W}}_{\mathrm{i} 1}$ & $(65,70,75)$ & $(65,70,75)$ & $(65,70,75)$ & $(65,70,75)$ & $(65,70,75)$ & $(65,70,75)$ & $(65,70,75)$ & $(65,70,75)$ \\
$\tilde{\mathrm{W}}_{\mathrm{i} 2}$ & $\tilde{\mathrm{W}}_{\mathrm{i} 1}-10$ & $\tilde{\mathrm{W}}_{\mathrm{i} 1}-10$ & $\tilde{\mathrm{W}}_{\mathrm{i} 1}-10$ & $\tilde{\mathrm{W}}_{\mathrm{i} 1}-10$ & $\tilde{\mathrm{W}}_{\mathrm{i} 1}-10$ & $\tilde{\mathrm{W}}_{\mathrm{i} 1}-10$ & $\tilde{\mathrm{W}}_{\mathrm{i} 1}-10$ & $\tilde{\mathrm{W}}_{\mathrm{i} 1}-10$ \\
$\tilde{\mathrm{W}}_{\mathrm{i} 3}$ & $\tilde{\mathrm{W}}_{\mathrm{i} 1}-20$ & $\tilde{\mathrm{W}}_{\mathrm{i} 1}-20$ & $\tilde{\mathrm{W}}_{\mathrm{i} 1}-20$ & $\tilde{\mathrm{W}}_{\mathrm{i} 1}-20$ & $\tilde{\mathrm{W}}_{\mathrm{i} 1}-20$ & $\tilde{\mathrm{W}}_{\mathrm{i} 1}-20$ & $\tilde{\mathrm{W}}_{\mathrm{i} 1}-20$ & $\tilde{\mathrm{W}}_{\mathrm{i} 1}-20$ \\
$\tilde{\mathrm{W}}_{\mathrm{i} 4}$ & $\tilde{\mathrm{W}}_{\mathrm{i} 1}-30$ & $\tilde{\mathrm{W}}_{\mathrm{i} 1}-30$ & $\tilde{\mathrm{W}}_{\mathrm{i} 1}-30$ & $\tilde{\mathrm{W}}_{\mathrm{i} 1}-30$ & $\tilde{\mathrm{W}}_{\mathrm{i} 1}-30$ & $\tilde{\mathrm{W}}_{\mathrm{i} 1}-30$ & $\tilde{\mathrm{W}}_{\mathrm{i} 1}-30$ & $\tilde{\mathrm{W}}_{\mathrm{i} 1}-30$ \\
$\mathrm{q}_{\mathrm{i} 1}$ & 150 & 150 & 150 & 150 & 150 & 150 & 150 & 150 \\
$\mathrm{q}_{\mathrm{i} 2}$ & 250 & 250 & 250 & 250 & 150 & 150 & 150 & 150 \\
$\mathrm{q}_{\mathrm{i} 3}$ & 350 & 350 & 350 & 350 & 250 & 250 & 250 & 250 \\
$\mathrm{SL}_{\mathrm{i}}$ & 0.5 & 0.6 & 0.6 & 0.5 & 0.5 & 0.6 & 0.6 & 0.5 \\
$\mathrm{P}_{\mathrm{i}}$ & 100 & 100 & 100 & 100 & 150 & 150 & 150 & 150 \\
$\mathrm{D}_{\mathrm{i}}$ & 10 & 10 & 10 & 10 & 10 & 10 & 10 & 10 \\
\hline
\end{tabular}


Table 2: Parameters of exponential and uniform distributions

\begin{tabular}{lllllllll}
\hline Product & 1 & 2 & 3 & 4 & 5 & 6 & 7 & 8 \\
\hline$\lambda_{\mathrm{i}}$ & $1 / 30$ & $1 / 30$ & $1 / 60$ & $1 / 60$ & $1 / 30$ & $1 / 30$ & $1 / 60$ & $1 / 60$ \\
$\mathrm{~T}_{\text {Min }_{\mathrm{i}}}$ & 20 & 20 & 50 & 50 & 20 & 20 & 50 & 50 \\
$\mathrm{~T}_{\mathrm{Max}_{\mathrm{i}}}$ & 40 & 40 & 70 & 70 & 40 & 40 & 70 & 70 \\
\hline
\end{tabular}

Table 3: The parameters and the best combination of the GA method The best combination for

\begin{tabular}{llllll} 
& & & & & $\begin{array}{l}\text { Uniform } \\
\text { distribution }\end{array}$ \\
Parameter & Alternatives & & $\begin{array}{l}\text { Exponential } \\
\text { distribution }\end{array}$ \\
\hline $\mathrm{P}_{\mathrm{c}}$ & 0.80 & 0.85 & 0.9 & 0.85 & 0.9 \\
$\mathrm{P}_{\mathrm{m}}$ & 0.076 & 0.098 & 0.1 & 0.098 & 0.098 \\
$\mathrm{~N}$ & 10 & 100 & 500 & 100 & 500 \\
\hline
\end{tabular}

Table 4: The best result for $\mathrm{R}_{\mathrm{i}}$

\section{Product}

$\begin{array}{lllllllllll}\text { Distribution } & 1 & 2 & 3 & 4 & 5 & 6 & 7 & 8 & \mathrm{Z}(\mathrm{R}, \tilde{\mathrm{W}}, \tilde{\mathrm{h}})\end{array}$

\begin{tabular}{llllllllll}
\hline Uniform & 305 & 344 & 622 & 635 & 358 & 335 & 1358 & 662 & 869320
\end{tabular}

$\begin{array}{llllllllll}\text { Exponential } & 213 & 283 & 416 & 550 & 231 & 280 & 416 & 553 & 113310\end{array}$

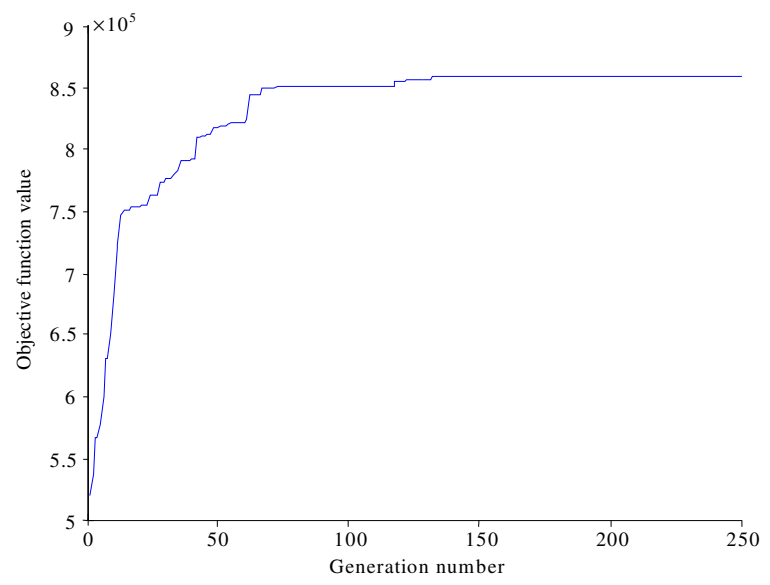

Fig. 4: The convergence path of the best result in uniform example

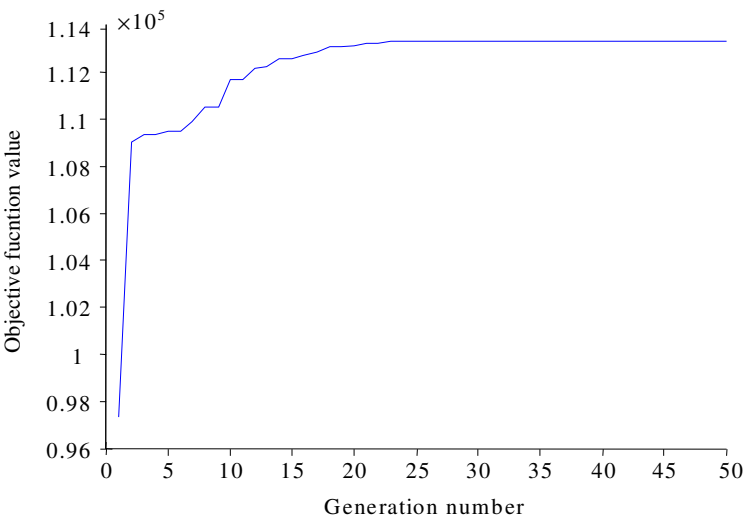

Fig. 5: The convergence path of the best result in exponential example

\section{CONCLUSION AND RECOMMENDATIONS FOR FUTURE RESEARCH}

In this research, a stochastic replenishment multiproduct inventory model with discount and fuzzy purchasing price and holding cost was investigated. Two mathematical modeling for two cases of uniform and exponential distribution of the time between two replenishments in case of incremental discount have been developed and shown to be fuzzy mixed integernonlinear programming problems. Then, a hybrid intelligent algorithm (fuzzy simulation+GA) has been proposed to solve the fuzzy integer non-linear problems.

Some recommendations for future works are (1) considering demands as fuzzy or random variables, (2) employing a total discount policy and (3) applying some other meta-heuristic algorithms.

\section{REFERENCES}

1. Chiang, C., 2003. Optimal replenishment for a periodic review inventory system with two supply modes. Eur. J. Operat. Res., 149: 229-244.

2. Mohebbi, E. and M.J.M. Posner, 2002. Multiple replenishment orders in continuous-review inventory system with lost sales. Operat. Res. Lett., 30: 117-129.

3. Feng, K. and U.S. Rao, 2007. Echelon-stock (RnT) control in two stage serial stochastic inventory system. Operat. Res. Lett., 35: 95-104.

4. Ouyang, L.Y. and B.R. Chuang, 2000. A periodic review inventory model involving variable leadtime with a service level constraint. Int. J. Syst. Sci., 31: 1209-1215.

5. Chiang, C., 2006. Optimal ordering policies for periodic-review systems with replenishment cycles. Eur. J. Operat. Res., 170: 44-56.

6. Qu, W.W., J.H. Bookbinder and P. Iyogun, 1999. An integrated inventory-transportation system with modified periodic policy for multiple products. Eur. J. Operat. Res., 115: 254-269.

7. Eynan, A. and D. Kropp, 2007. Effective and simple EOQ-like solutions for stochastic demand periodic review systems. Eur. J. Operat. Res., 180: 1135-1143.

8. Bylka, S., 2005. Turnpike policies for periodic review inventory model with emergency orders. Int. J. Prod. Econ., 93-94: 357-373.

9. Mohebbi, E., 2004. A replenishment model for the supply-uncertainty problem. Int. J. Econ., 87: $25-37$. 
10. Taleizadeh, A.A., M.B. Aryanezhad and S.T.A. Niaki, 2008. Optimizing multi-product multi-constraint inventory control systems with stochastic replenishments. J. Applied Sci., 8: $1228-1234$.

11. Hsieh, C.H., 2002. Optimization of fuzzy production inventory models. Inform. Sci., 146: 29-40.

12. Roy, A., M.K. Maiti, S. Kar and M. Maiti, 2007. Two storage inventory model with fuzzy deterioration over a random planning horizon. Math. Comput. Modeling, 46: 1419-1433.

13. Yao, J., S. Chang and J. Su, 2000. Fuzzy inventory without backorder for fuzzy order quantity and fuzzy total demand quantity. Comput. Operat. Res., 27: 935-962.

14. Das, K., T.K. Roy and M. Maiti, 2004. Multi-item stochastic and fuzzy-stochastic inventory models under two restrictions. Comput. Operat. Res., 31: 1793-1806.

15. Chang, H.C., J.S.H. Yao and L.Y. Ouyang, 2004. Fuzzy mixture inventory model with variable lead time based on probabilistic fuzzy set and triangular fuzzy number. Math. Comput. Modeling, 39: 287-304.
16. Mandal, N.K. and T.K. Roy, 2006. A displayed inventory model with l-r fuzzy number. Fuzzy Optimization Decision Making, 5: 227-243.

17. Maiti, M.K. and M. Maiti, 2006. Fuzzy inventory model with two warehouses under possibility constraint. Fuzzy Sets Syst., 157: 52-73.

18. Liu, S.T., 2007. Fuzzy profit measures for a fuzzy economic order quantity model. To Appear in the J. Applied Math. Modeling.

19. Liu, B., 2004. Uncertainty Theory: An Introduction to Its Axiomatic Foundations. Springer, Berlin.

20. Ertogal, K. and M.A. Rahim, 2005. Replenish-upto inventory control policy with random replenishment interval. Int. J. Prod. Econ., 93- 94: 399-405.

21. Gen, M., 1997. Genetic Algorithm and Engineering Design. John Wiley \& Sons, New York, NY, USA.

22. Holland, J.H., 1975. Adoption in neural and artificial systems. The University of Michigan Press, Ann Arbor, Michigan.

23. Man, K.F., K.S. Tang, S. Kwong and W.A. Halang, 1997. Genetic Algorithms for Control and Signal Processing. Springer Verlag, London. 